\title{
Design Luenberger Observer for an Electromechanical Actuator
}

\author{
Zs. Horváth, Gy. Molnárka \\ Department of Mechatronics and Machine Design \\ Széchenyi István University, Egyetem tér 1, H-9026, Győr, Hungary \\ zsolt2.horvath@audi.hu
}

Abstract: In this work we have designed a Luenberger Observer for an Electromechanical Actuator (EMA). We have considered a simple linear model of the Actuator plant with neglecting nonlinearities. The model of the EMA, the Observer and the simulations have been computed in MATLAB ${ }^{\circledR}$. In our simulation we have examined the dynamics of the planned Observer. With different pole placement proportions and state estimation error conditions, three states have been estimated: Motor current $\left(X_{1}\right)$, angular velocity of the throttle plate $\left(X_{2}\right)$, and angular position of the throttle plate $\left(X_{3}\right)$. Outline of our examination should be to take the conclusions from the influence of the pole placement and error condition on the dynamics of the Observer. Thus determining of an optimal pole values for the proposed Luenberger Observer. Outline of this paper could be used for further research topics (fault detection in Electromechanical Actuator).

Keywords: observer, observability, EMA plant-model, state space equation, poleplacement

\section{Introduction}

With increasing number of processing integrated electromechanical systems on current automotive vehicles, such as actuators, sensors and microcomputers, the field of supervision (monitoring), diagnosis and control plays an inportant role. Electromechanical Actuators often have to perform in extreme plant conditions, measurement of needed signals is often not possible or it is too expensive, and that is why their state control and diagnostic is important.

In applications feedback control or diagnosis systems the entire state vector of the system must be controlled. If the state vector can not be measured, what is a tipical case in most complex systems, one suitable approximation to the state vector is needed. That can be substituted into a control law. A system which produces such an approximation, is called an Observer or Luenberger Observer. It was Luenberger [1] who has first 
developed an Observer for linear control systems, and after that it was introduced for nonlinear systems too, by Thau [2].

This paper proposes a design for a Luenberger Observer for an electromechanical throttle valve. Many different observer concepts are nowadays succesfully used for modelling and control of Electromechanical Actuators. Nonlinear variable structure system (VSS) observer for throttle systems are presented in e.g. [8]. Paper [3] uses a sliding-mode observer for a robust position control of these Actuators. The most common approaches are using Extended Kalman Filter (EKF) or Unscented Kalman Filter (UKF) [7], which are preferable solutions when the process nonlinearities are strong and noise is associated with the real system. Another study [5] proposes using of the Kalman Filter for the observation of the DC-motor. A further study is applying Luenberger Observer for Sensor Monitoring in Active Front Steering Systems can be found in [11].

The throttle valve is a type of an Electromechanical Actuator, which is advanced in several applications of combustion engine control (intake manifold, exthaust gas recirculation, variable turbine geometry, ect.). The simple EMA plant model is a system of 3 rd order.

The presented paper consists of : in Section I the EMA plant model is introduced, then in Section II the fundamentals of Luenberger Observer are revised, in Section III the design of Luenberger Observer is described. Finally in Section IV the main obtained results are presented in this paper.

\section{Model of an Electromechanical Actuator}

The simple model of the EMA Figure 1. consists of following parts: DC-motor, gearbox, return spring, throttle plate and sensor for valve position. The DC-motor is supplied with a bipoloar chopper, motor shaft rotation is transmitted through a gearbox to the throttle plate. The position of the throttle plate is given to the ECU (Engine Control Unit). A reset spring places the de-energized throttle plate in its full open position. The position control of the throttle valve and the diagnosis of the actuator is done by the engine ECU (ECU will be not examined in this work).

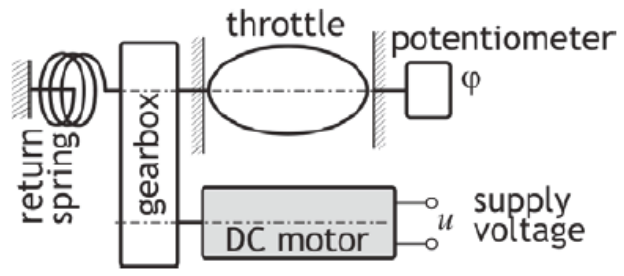

Figure 1. The scheme of the Electromechanical Actuator (EMA) [6]

\subsection{Model Equations of the EMA}

The relation between input voltage $u_{a}$ and current $i$ in the armature circuit can be described as 


$$
u_{a}=R i+L \frac{d i}{d t}+K_{e} n \omega
$$

where $L$ is the inductance, $R$ is the resistance in the armature circuit, $K_{e}$ is the inductive voltage constant, $n$ is the gear ratio and $\omega$ is the angular velocity of the throttle plate .

The motion Equations of the throttle plate (related to the plate axel) can be desribed as

$$
J \frac{d \omega}{d t}=K_{t} i-M_{o}-c \varphi-M_{c} \operatorname{sign}(\omega)-K_{V} \omega
$$

where $\varphi$ is the angular position of the throttle plate, $\mathrm{J}$ is the rotary inertia at the throttle shaft, which is composed of inertia of the DC-motor. Mo denotes a torque due to the prestressed springs, $\mathrm{c}$ is a linear spring constant. Mc is a torque of the coulomb friction of the system. $\mathrm{Kv}$ is a positive constant for viscousus friction. Finally $\mathrm{Kt}$ denotes the motor torque constant.

Table 1. EMA parameters

\begin{tabular}{|c|c|c|}
\hline Parameter & Values & Units \\
\hline $\mathrm{R}$ & 4.3 & $\mathrm{Ohm}$ \\
\hline $\mathrm{L}$ & 0.002 & $\mathrm{H}$ \\
\hline $\mathrm{K}_{\mathrm{e}}$ & 0.017 & $\mathrm{Vs} / \mathrm{rad}$ \\
\hline $\mathrm{K}_{\mathrm{t}}$ & 0.017 & $\mathrm{Nm} / \mathrm{A}$ \\
\hline $\mathrm{J}$ & 0.0016 & $\mathrm{kgm}$ \\
\hline $\mathrm{n}$ & 40 & - \\
\hline $\mathrm{M}_{\mathrm{c}}$ & 0.075 & $\mathrm{Nm}$ \\
\hline $\mathrm{M}_{0}$ & 0.25 & $\mathrm{Nm}$ \\
\hline $\mathrm{c}$ & 0.059 & $\mathrm{Nm} / \mathrm{rad}$ \\
\hline $\mathrm{K}_{\mathrm{v}}$ & 0.02 & $\mathrm{Nms} / \mathrm{rad}$ \\
\hline $\mathrm{K}_{\mathrm{s}}$ & 2.73 & $\mathrm{~V} / \mathrm{rad}$ \\
\hline
\end{tabular}

Considering the Equations (1) and (2), written down in the standard state-space description and neglecting the nonlinearities of the Coulomb friction (we are controlling the valves only in one direction now), the input voltage (ua) is $12 \mathrm{~V}$, the measured variable is the angular position of the plate $\varphi \in\left(0^{\circ} \ldots 90^{\circ}\right)$.

For a linear time-invariant process the state space Equations can be given as

$$
\begin{aligned}
& \dot{x}=A x+B u \\
& y=C^{T} x+D^{T} u
\end{aligned}
$$

with $u \in \mathbf{R}^{r}, x \in \mathbf{R}^{n}, y \in \mathbf{R}^{m}$

The elements of the Equations are the state vector $\mathrm{x}(\mathrm{t})$, input $\mathrm{u}(\mathrm{t})$, output $\mathrm{y}(\mathrm{t})$, state matrix A, input vector B, output vector $\mathrm{CT}$ and the direct feedthrough $\mathrm{D}$. 
The first Equation here is the state Equation and the second is the output.

With defining the state vector and input vector, it can be written as

$$
x=\left[\begin{array}{l}
x_{1} \\
x_{2} \\
x_{3}
\end{array}\right]=\left[\begin{array}{l}
i \\
\omega \\
\varphi
\end{array}\right] ; u=\left[\begin{array}{c}
u_{a} \\
m_{t} \\
0
\end{array}\right]
$$

Where $x_{1}$ is the first component of the state vector $x$, and it represents the motor current, $x_{2}$ is the angular velocity, and $x_{3}$ is the angular position of the throttle plate.

In the Equation of the input vector are the input voltage and the sum torque of the Coulomb friction and spring pretension (they are constant).

For the state space description of our model, Equations (1) and (2) are brought into the form

$$
\begin{aligned}
& \frac{d i}{d t}=-\frac{R}{L} i-n \frac{K e}{L} \omega+\frac{1}{L} u_{a} \\
& \frac{d \omega}{d t}=n \frac{K_{t}}{J} i-\frac{K_{v}}{J} \omega-\frac{M_{c}+M_{0}}{J}-\frac{c}{J} \varphi
\end{aligned}
$$

Eq. (5) written into a form vector-matrix

$$
\begin{aligned}
& {\left[\begin{array}{l}
\dot{x}_{1} \\
\dot{x}_{2} \\
\dot{x}_{3}
\end{array}\right]=\left[\begin{array}{ccc}
-\frac{R}{L} & -n \frac{K_{e}}{L} & 0 \\
n \frac{K_{t}}{J} & -\frac{K_{v}}{J} & -\frac{c}{J} \\
0 & 1 & 0
\end{array}\right]\left[\begin{array}{c}
x_{1} \\
x_{2} \\
x_{3}
\end{array}\right]+\left[\begin{array}{ccc}
\frac{1}{L} & 0 & 0 \\
0 & -\frac{1}{J} & 0 \\
0 & 0 & 0
\end{array}\right]\left[\begin{array}{c}
u_{a} \\
m_{t} \\
0
\end{array}\right]} \\
& y=\left[\begin{array}{lll}
0 & 0 & K_{s}
\end{array}\right]\left[\begin{array}{l}
x_{1} \\
x_{2} \\
x_{3}
\end{array}\right]+\left[\begin{array}{lll}
0 & 0 & 0
\end{array}\right]\left[\begin{array}{c}
u_{a} \\
m_{t} \\
0
\end{array}\right]
\end{aligned}
$$

Through substituting the parameter values of the EMA into the Eq. (6) we receive the following state space model of the plant 


$$
\begin{aligned}
& {\left[\begin{array}{l}
\dot{x}_{1} \\
\dot{x}_{2} \\
\dot{x}_{3}
\end{array}\right]=\left[\begin{array}{ccc}
-2150 & -340 & 0 \\
420 & -12.5 & -36.7 \\
0 & 1 & 0
\end{array}\right]\left[\begin{array}{l}
x_{1} \\
x_{2} \\
x_{3}
\end{array}\right]+\left[\begin{array}{ccc}
500 & 0 & 0 \\
0 & -625 & 0 \\
0 & 0 & 0
\end{array}\right]\left[\begin{array}{c}
u_{a} \\
0.32 \\
0
\end{array}\right]} \\
& y=\left[\begin{array}{lll}
0 & 0 & 2.73
\end{array}\right]\left[\begin{array}{l}
x_{1} \\
x_{2} \\
x_{3}
\end{array}\right]+\left[\begin{array}{lll}
0 & 0 & 0
\end{array}\right]\left[\begin{array}{c}
u_{a} \\
0.32 \\
0
\end{array}\right]
\end{aligned}
$$

as well as the transfer functions for angular position

$$
\begin{aligned}
& W_{1}(s)=\frac{0.68}{3.2 \cdot 10^{6} s^{3}+6.92 \cdot 10^{-3} s^{2}+0.543 s+0.252} \\
& W_{2}(s)=-\frac{2 \cdot 10^{-3} s+4.3}{3.2 \cdot 10^{6} s^{3}+6.92 \cdot 10^{-3} s^{2}+0.543 s+0.252} \\
& \mathrm{~W}(\mathrm{~s})=W_{1}(\mathrm{~s})+W_{2}(s)
\end{aligned}
$$

The angular position of the plate can be determined as

$$
\begin{aligned}
& \varphi(\mathrm{s})=W_{1}(\mathrm{~s}) \mathrm{u}_{a}(\mathrm{~s})+W_{2}(s) \mathrm{m}_{t} \\
& \text { where } \\
& m_{t}=M_{0}+M_{c}
\end{aligned}
$$

$m_{t}$ is a load torque during the plate opening.

The parameters of the plant are shown in the Table 1.

Fig. 2 shows the step response of second and third state, $\omega, \varphi$ respectively by input voltage of $12 \mathrm{~V}$.
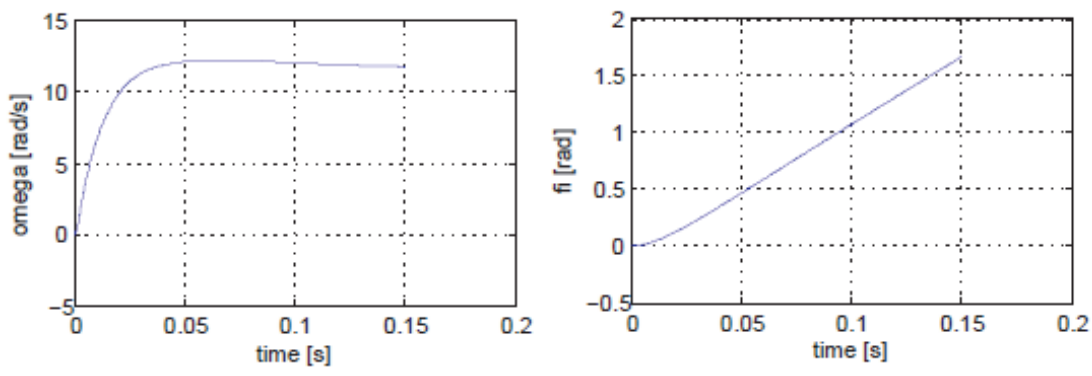

Figure 2. Step responses of the EMA

left: Angular velocity of throttle plate, right: Angular posion of throttle plate

With getting the step responses, we are able to see the linear process dynamics of the EMA. The left side of Figure 2 shows the high dynamics of the EMA with a settling 
time of $\sim 0.05 \mathrm{sec}$. We must take this in consideration by designing the Observer when determining the poles.

\section{Luenberger Observer}

Luenberger was the first who suggested the state Observer for estimating the unknown states of a deterministic linear system in 1971 [1]. The Equation for this contains a correction term for current state estimates, which corrects with an amount that is proportional to the error that we predicted: reducing the current output sum with the actually measured sum. With this output sum reduction we can ensure the stability and convergence of the Observer even in case of unstable state of the observed system.

\subsection{Observability of Time-Invariant Systems}

Observability is a necessary condition for investigating an Observer.

Observability [2] is the issue of whether the state of dynamic system with a known model is uniquely determinable from its inputs and outputs. It is essentially a property of the given system model. A given linear dynamic system model with a given linear input/output model is considered observable if and only if its state is uniquely determinable from the models definition, its inputs and outputs.

The observability of time invariant systems can be characterised with the rank of the observability matrices.

As already mentioned in section 2.1, the elements of the Equation (10) are:

- $\quad \boldsymbol{A}$ : state matrix

- $\quad C^{T}$ : output vector

$$
M_{0}=\left[\begin{array}{c}
C^{T} \\
C^{T} A \\
\vdots \\
C^{T} A^{n-1}
\end{array}\right]
$$

for continuous-time systems.

The systems are observable if the dimension of the system state vector is rank $\mathrm{n}$. The observability matrix for the EMA, using Equation (10) is

$$
M_{0}=\left[\begin{array}{ccc}
0 & 0 & 2.7 \\
0 & 2.7 & 0 \\
1146 & -34.1 & -100.3
\end{array}\right]
$$

Here, $M$ has a rank (rank=3) equal to the dimension of $x(t)$, therefore our system is observable. 


\subsection{Basic Theory of Luenberger Observer}

Considering the state space system again

$$
\begin{aligned}
& \dot{x}(t)=A x(t)+B u(t), \quad x(0)=0 \\
& y(t)=C x(t)
\end{aligned}
$$

with enlarging Eq. (12) through additional input sequence $u_{B}$

$$
\begin{aligned}
& \frac{d \hat{x}(t)}{d t}=A \hat{x}(t)+B u(t)+u_{B}(\mathrm{t}), \quad \hat{x}(0)=\hat{x}_{0} \\
& \hat{y}(t)=C \hat{x}(t)
\end{aligned}
$$

where

$$
u_{B}(t)=L(y(t)-\hat{y}(t))
$$

In designing the Observer, the matrices $A, B, C$ are fixed, but the $\mathrm{n} \mathrm{x}$ m matrix $L$ is arbitrary. $L$ is called as Observer gain. An identity Observer is determined by selection of $L$.

Through substituting Eq. (14) into Eq. (13) and taking in consideration that

$$
\begin{aligned}
& \hat{y}(t)=C \hat{x}(t) \\
& \text { and } \\
& y(t)=C x(t)
\end{aligned}
$$

the Equation (13) is brought into the form

$$
\frac{d \hat{x}(t)}{d t}=A \hat{x}(t)+B u(t)+L C(x(t)-\hat{x}(t))
$$

With the obtained state matrix of the Observer $[A-L C]$, the full Equation of the Luenberger Observer is

$$
\frac{d \hat{x}(t)}{d t}=(A-L C) \hat{x}(t)+B u(t)+L y(t)
$$

$u$ and $y$ are the inputs of the Observer, $\hat{x}(t)$ is an estimate of the plant state $x(t)$.

The estimation error is defined as

$$
e(t)=x(t)-\hat{x}(t)
$$


which is not directly measurable in most cases, because $x(t)$ is not available as an input to the obeserver.

With taking the first derivate of $e(t)$ we can get

$$
\begin{aligned}
& \dot{e}(t)=\frac{d}{d t}(x(t)-\hat{x}(t))=A x(t)+B u(t)-A \hat{x}(t)- \\
& -B u(t)-L C(x(t)-\hat{x}(t))=(A-L C)(x(t)-\hat{x}(t))
\end{aligned}
$$

Equation (19) has proved that the dinamics of the observing process is determined by matrix $[A-L C]$. The eigenvalues of $[A-L C]$ are satisfactory, the reason for this is that the Observer has to be able to imitate the plant state.

The dinamic of the error is given by

$$
\begin{aligned}
& \dot{e}(t)=(A-L C) e(t), \quad e(0)=x_{0}-\hat{x}_{0} \\
& \lim _{t \rightarrow \infty}\|e(t)\|=0
\end{aligned}
$$

To obtain the accurate state estimates with no regard of $e(0), e(t)$ as $t \rightarrow \infty$ exponentially, we must choose all the eigenvalues of the $[A-L C]$ matrix to lie in the left half s - plane. To achieve that, the state of the Observer converges to the state of the Observer system, the Observers eigenvalues must be negative, moreover, they should be a little more negative than the eigenvalues of the observed system. In that case the convergence is faster than other system effects. In theory, these eigenvalues can be moved to minus infinity arbitrarily, to achieve extreme fast convergence. But this leads the Observer to act like a differentiator, become very sensitive to noise and arise other difficulties. The problem of selecting good eigenvalues is still not totally resolved, but a functional practice is that we should place them so, that the Observer can be a little faster than the rest of the closed-loop system [4].

Figure 3 helps in the interpretation of the Luenberger Observer.

The elements of this Figure are:

- $\quad x$ : state vector

- $u$ : input

- $y:$ output

- $\quad \boldsymbol{A}$ : state matrix

- $\quad \boldsymbol{B}$ : input vector

- C : output vector

- L: Observer gain

- $\quad \boldsymbol{e}$ : estimation error 


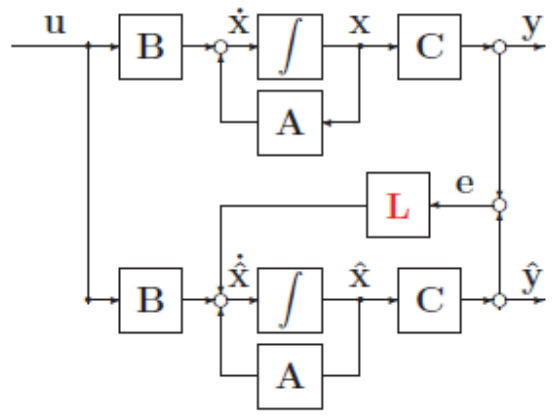

Figure 3. Scheme of the Luenberger Observer

The residual is the difference between the measured output and the actual observation. It can be written as

$$
r(t)=y(t)-\hat{y}(t)
$$

In ideal case is, when $r(t)=0$ during the entire operational interval if the Observer is a perfect model of the plant. However in reality there are uncertainities in the model of the plant represented by $\Delta A, \Delta B$ and $\Delta C$. These are neglected in this work. In case when an instrument fault occurs the estimated vector will be $\hat{x}(t)$ upset. That makes the value of the residual nonzero. As a result, the residual can be used also for fault detection of instrument faults.

\subsection{Design of the Luenberger Observer}

For designing an Observer we must know the dynamics of the observed system, defined by the eigenvalues of the system.

As known, the eigenvalues of the plant can be calculated with a characteristical polynom as follows

$$
|A-\lambda \cdot E|=-\lambda^{3}-\lambda^{2} \cdot 2162.5-\lambda \cdot 169711.25-77937.5
$$

from this, the poles of the plant are

$$
\lambda_{1}=-2081 ; \lambda_{2}=-81.1 ; \lambda_{3}=-50
$$

Knowing the poles of the observed system, the next step is defining the Observers poles [A-LC]. Generally the formula is that we define 2-6 times bigger poles for the Observer than the dominant systems poles [4]. The barrier for the bigger poleshifting is the increasing noise in the Observer. My goal in this work is setting up 5 times bigger poleshifting, since I calculate with small noise rate.

The reason for choosing factor 5 is that the eigenvalues of the $[\mathrm{A}-\mathrm{LC}]$ matrix needs to be prompt compared at beginning of the operating interval too. 
As we can see, the EMA plant has a small dominant time constant and therefore small settling-time.

From this the proposed eigenvalues of the Observer

$$
\begin{gathered}
\mathrm{P}_{0}=\operatorname{det}\left(s I-A+L c^{T}\right)=F(s)=s^{n}+f_{1} s^{n-1}+\ldots+f_{n-1} s+f_{n} \\
P_{0}=[-2331 ;-331.1 ;-250.5]^{T}
\end{gathered}
$$
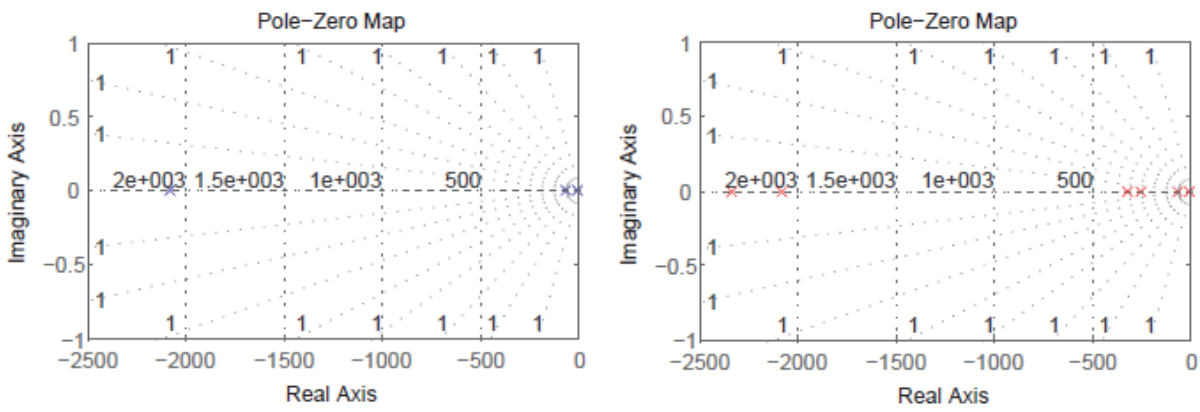

Figure 4. The pole placement for the Observer showed on the Pole-Zero Map left: poles of the EMA plant, right: desired poles of the Observer

The next step of the design is determining the feedback gain matrix $L$.

A number of algorithms can be found in the Literature [4], [10] to do that, some of which are built in a popular control system design software. In MATLAB ${ }$ commands acker or place can be used as feedback to calculate the matrix L.

If the plant model is given in transfer function form, the poles can be easily taken with using the state space canonical form. In the matrix of the canonical form the poles of the plant are placed in the first column ( Eq. (25) ).

$$
\begin{aligned}
A_{0} & =\left[\begin{array}{ccccc}
-a_{1} & 1 & 0 & \cdots & 0 \\
-a_{2} & 0 & 1 & \cdots & 0 \\
\vdots & \vdots & \vdots & \ddots & \vdots \\
-a_{n-1} & 0 & 0 & \cdots & 1 \\
-a_{n} & 0 & 0 & \cdots & 0
\end{array}\right] ; \\
C_{0}^{T} & =\left[\begin{array}{cccc}
1, & 0, & \ldots, & 0
\end{array}\right] ; b_{0}=\left[\begin{array}{llll}
b_{1}, & b_{2}, & \ldots, & b_{n}
\end{array}\right]^{T}
\end{aligned}
$$

To determine the Observer feedback matrix in canonical form, the matrix Equation can be described as 


$$
\begin{aligned}
& A_{0}-L_{0} C^{T}=\left[\begin{array}{ccccc}
-a_{1} & 1 & 0 & \cdots & 0 \\
-a_{2} & 0 & 1 & \cdots & 0 \\
\vdots & \vdots & \vdots & \ddots & \vdots \\
-a_{n-1} & 0 & 0 & \cdots & 1 \\
-a_{n} & 0 & 0 & \cdots & 0
\end{array}\right]- \\
& -L_{0}\left[\begin{array}{llllll}
1, & 0, & \ldots, & 0
\end{array}\right]=\left[\begin{array}{ccccc}
-f_{1} & 1 & 0 & \cdots & 0 \\
-f_{2} & 0 & 1 & \cdots & 0 \\
\vdots & \vdots & \vdots & \ddots & \vdots \\
-f_{n-1} & 0 & 0 & \cdots & 1 \\
-f_{n} & 0 & 0 & \cdots & 0
\end{array}\right] \\
& L=L_{0}=\left[f_{1}-a_{1}, f_{2}-a_{2}, \ldots, f_{n}-a_{n}\right]^{T}
\end{aligned}
$$

From this the Observer feedback matrix is

$$
L=[719670 ;-129350 ; 270]^{T}
$$

The Observer gain matrix can be written as follows:

$$
\begin{aligned}
& A-L C= \\
& {\left[\begin{array}{ccc}
-2150 & -340 & 0 \\
420 & -12.5 & -36.7 \\
0 & 1 & 0
\end{array}\right]-\left[\begin{array}{ccc}
0 & 0 & 1964700 \\
0 & 0 & -353100 \\
0 & 0 & 800
\end{array}\right]} \\
& =\left[\begin{array}{ccc}
-2200 & -300 & -1964700 \\
400 & 0 & 353100 \\
0 & 0 & -800
\end{array}\right]
\end{aligned}
$$

Finally the state space Equation of the Luenberger Observer resulted from Eq. (28) is determined to be:

$$
\begin{aligned}
& \frac{d \hat{x}}{d t}=\left[\begin{array}{ccc}
-2200 & -300 & -1964700 \\
400 & 0 & 353100 \\
0 & 0 & -800
\end{array}\right] x+ \\
& +\left[\begin{array}{ccc}
500 & 0 & 0 \\
0 & -625 & 0 \\
0 & 0 & 0
\end{array}\right] u+\left[\begin{array}{c}
719670 \\
-129350 \\
270
\end{array}\right] y
\end{aligned}
$$




\section{Experimental Results}

In the following I will examine the state variables of the EMA model and the function of the Observer group planned for it, with different pole placement proportions and state estimation error conditions. Outline of our examination should be to take the conclusion to the influence of the pole placement and error condition on the dynamics of the observer. The algorithm for the Observer has been computed in MATLAB ${ }^{\circledR}$. The throttle valve is excited with a step input signal, the input voltage is $12 \mathrm{~V}$. Three states have been estimated in the simulation: Motor current (X1), angular velocity of the throttle plate (X2), and angular position of the throttle plate (X3). The pole placement factor is calculated as follows: $k=P_{d o m}+P_{0}$.

In the first case the pole placement rate $(\mathrm{k}=-50)$ and value of the estimation error condition were small $\left(e_{0}=[0.1,0.1,0.4]\right)$, showed on the Figure 5 .

The Observer follows the state variables of the EMA plant with slow dynamics. This doesn't fulfill the dynamic requirements.
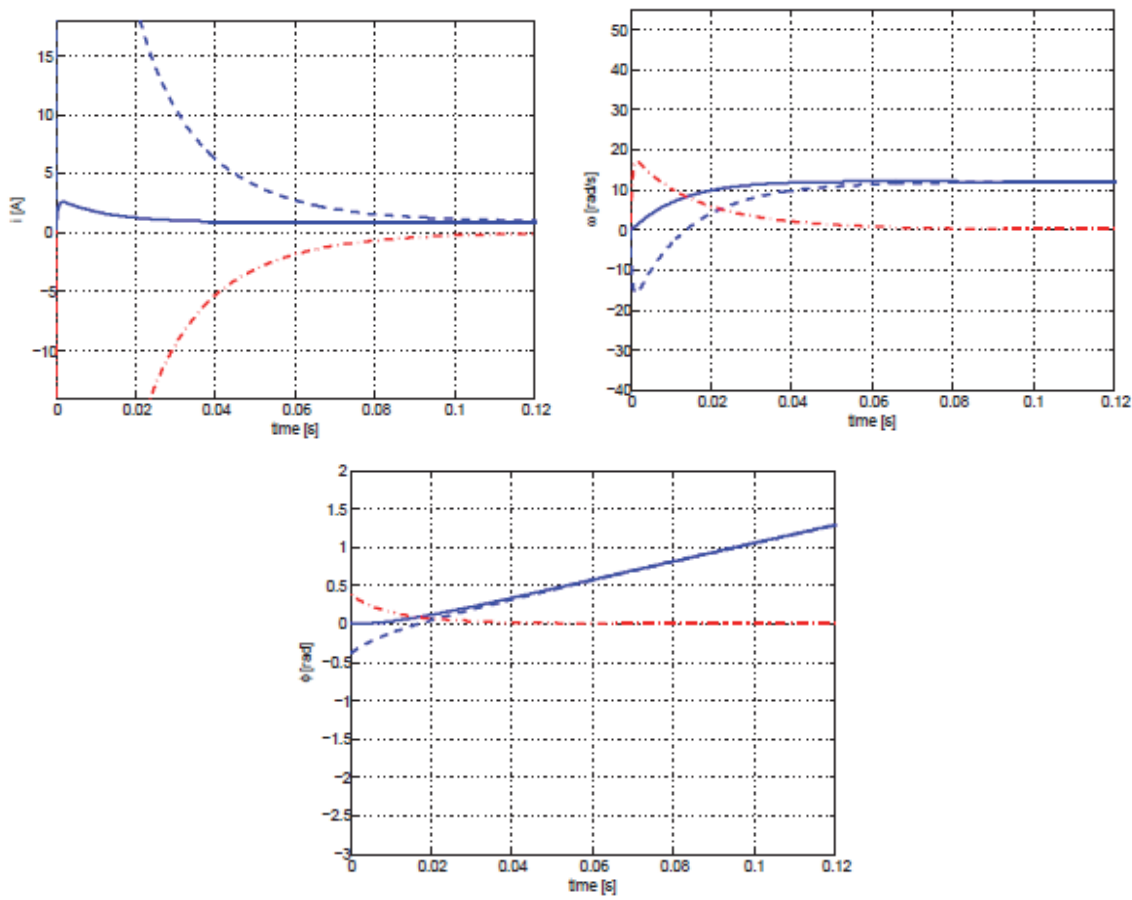

Figure 5. States of the EMA model with estimated pole placement rate of $k=-50$ and setting small estimation error condition ( $e_{0}=[0.1,0.1,0.4]$ )

blue : state of the plant, dashed blue : estimated state, d.-dashed red : estimation error top left: motor current, top right: angular velocity, bottom : angular position of the plate 
In the second case the pole placement rate $(k=-50)$ and the value of the estimation error condition were big $\left(e_{0}=[0.1,0.1,2.5]\right)$, as showed on the Figure 6 .

The Observer follows the state variables of the EMA plant with slower dynamics than in the first case. That is because of the big estimation error condition. These pole settings doesn't fulfill the dynamic requirements.
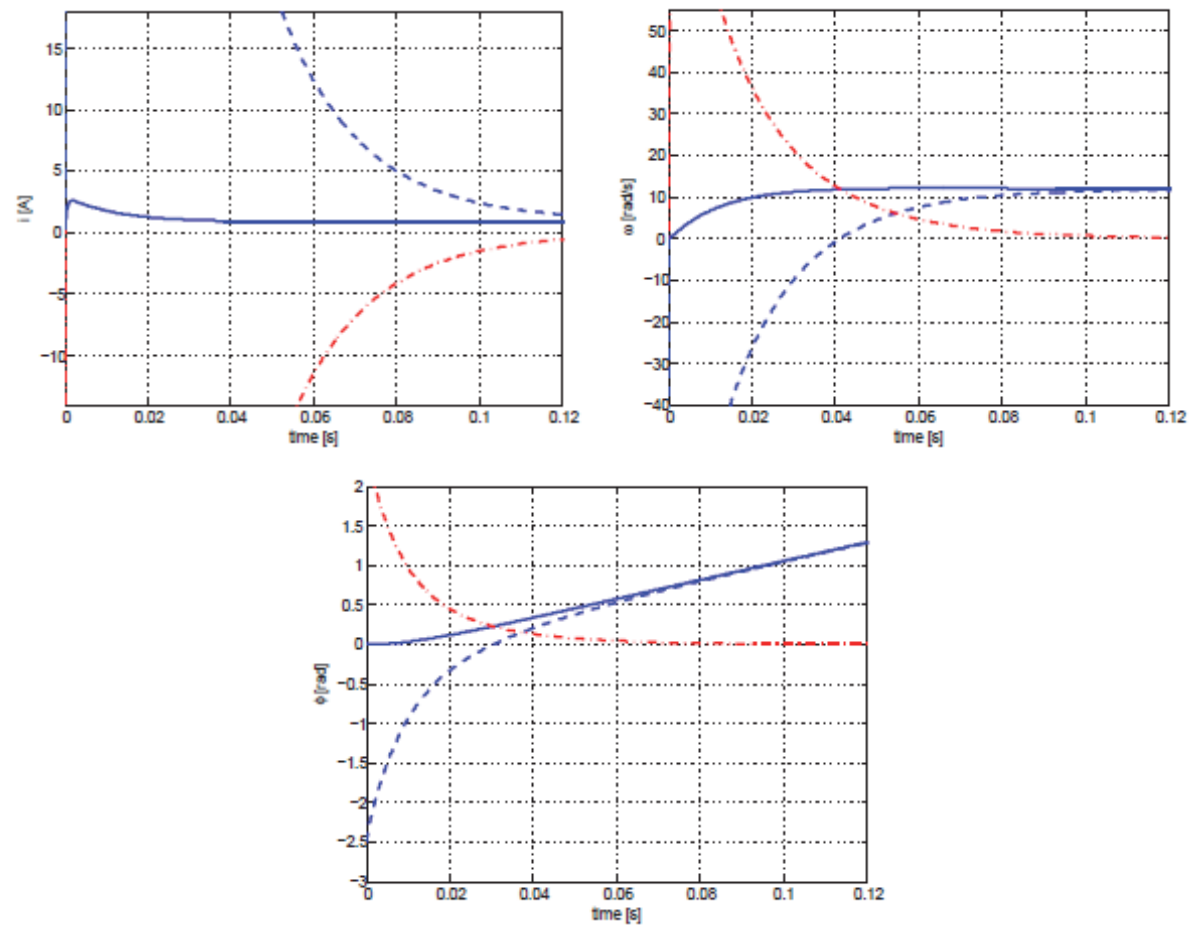

Figure 6. States of the EMA model with estimated pole placement rate of $k=-50$ and setting big estimation error condition ( $e_{0}=[0.1,0.1,2.5]$ )

blue : state of the plant, dashed blue : estimated state, d.-dashed red : estimation error top left: motor current, top right : angular velocity, bottom : angular position of the plate

After this, we have increased the pole placement rate $(k=-100)$. The value of the estimation error condition was small $\left(\mathrm{e}_{0}=[0.1,0.1,0.4]\right)$, as showed on the Figure 6 . We have experienced, that the Observer follows the state variables of the EMA plant with satisfactory dynamics. This would fulfill the dynamic requirements.

But when the pole placement rate $(\mathrm{k}=-100)$ stayed the same, and the value of the estimation error condition has been increased to big $\left(e_{0}=[0.1,0.1,2.5]\right.$, the Observer follows the state variables of the EMA plant with slower dynamics than in the first case. The estimated state variables of motor current and angular velocity of the throttle plate needed more time to settle than in the case with small estimation error condition. These pole settings doesn't fulfill the dynamic requirements either. 
As a result from these, it can be stated, that as far as the estimation error condition is increasing, the Observers settling time will be slower and slower.

In the case when the pole placement rate $(\mathrm{k}=-200)$ and the value of the estimation error condition were small $\left(e_{0}=[0.1,0.1,0.4]\right)$, we have experienced that the Observer follows the state variables of the EMA plant with adequate dynamics. The settling time was short for every estimated state. This fulfills the dynamic requirements.

In the last case the pole placement rate was the same $(\mathrm{k}=-200)$, but the value of the estimation error condition was big $\left(e_{0}=[0.1,0.1,2.5]\right)$, as shown on the Figure 7 . The Observer follows the state variables of the EMA plant with a little slower dynamics than in the first case. The estimated state variables of motor current and angular velocity of the throttle plate needed more time for settling on than in the case with small estimation error condition. In spite of this, this pole settling fulfills the dynamic requirements.

As a result from these, it can be stated, that the placement rate $\mathrm{k}=-200$ had to fulfill the dynamic requirements observing the throttle valve in every condition.
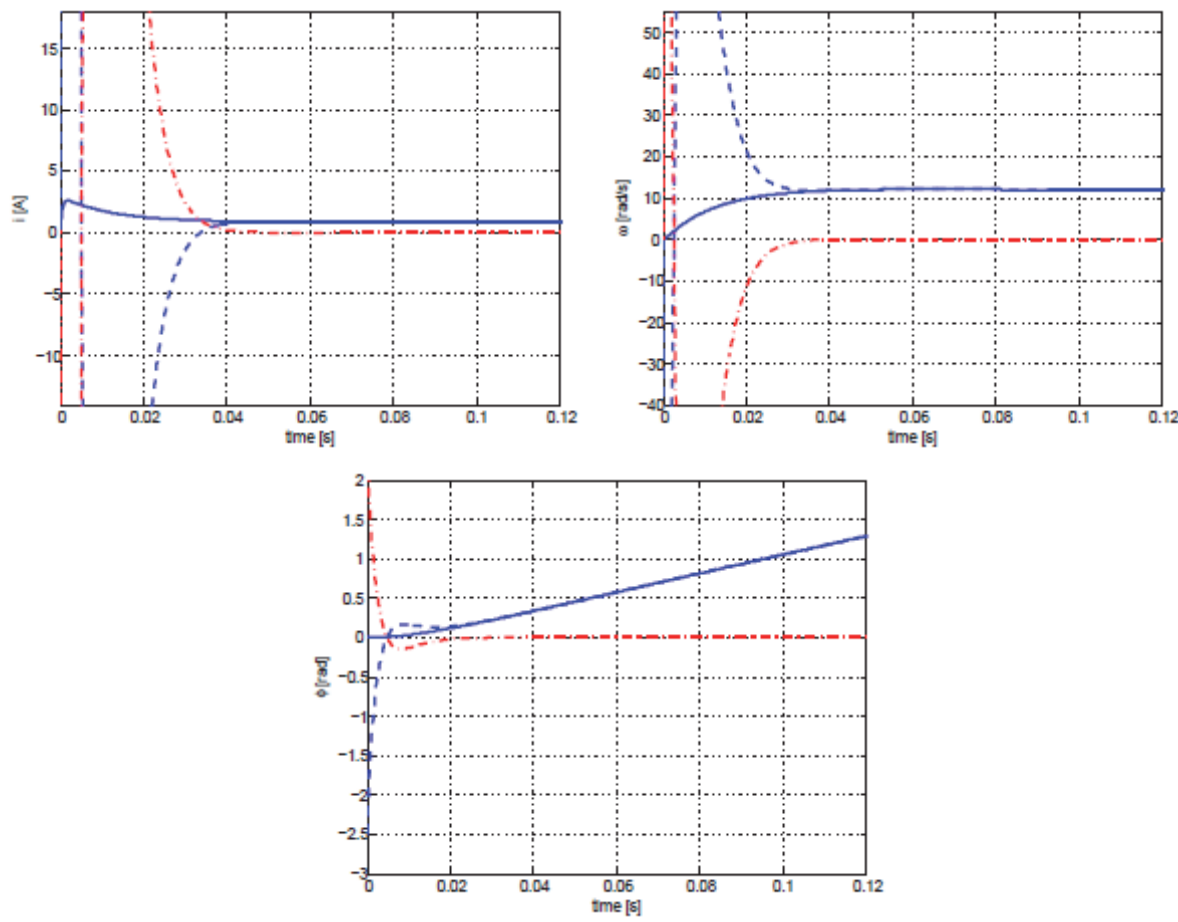

Figure 7. States of the EMA model with estimated pole placement rate of $k=-200$ and setting big estimation error condition ( $\left.e_{0}=[0.1,0.1,2.5]\right)$

blue : state of the plant, dashed blue : estimated state, d.-dashed red : estimation error top left: motor current, top right : angular velocity,

bottom : angular position of the plate 


\section{Conclusion}

This paper demonstrates a design of the Luenberger Observer for an Electromechanical Actuator. For this design, we have considered a simple linear model of the EMA plant with neglecting nonlinearities. The models of the EMA and the Observer were

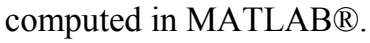

In the simulation we have examined the dynamics of the Observer to be planned for it.

With different pole placement proportions and state estimation error conditions three states have been estimated: Motor current (X1), angular velocity of the throttle plate (X2), and angular position of the throttle plate (X3).

In the last case of the simulation with pole placement rate of $(\mathrm{k}=-200)$, despite the value of the estimation error condition was big $\left(e_{0}=[0.1,0.1,2.5]\right)$, the Observer has fulfilled the dynamic requirements. It can be stated, that the placement rate $\mathrm{k}=-200$ has to fulfill the dynamic requirements observing the throttle valve in every condition.

As a result of our examination, we can state two things:

Firstly, the more the pole of the Observer is negative placed in the left direction, the Observers settling time will be shorter and shorter. Secondly, as far as the estimation error condition is increasing, the Observers settling time will be slower and slower. With setting the eigenvalues 5 times bigger for the Observer than the eigenvalues of the dominant system, it could be achieved that the convergence of the estimation is faster than others system effects.

Outline of this work is, despite that Luenbergers Observer is not a new method, it gives a simple algorithm to state space observing of Electromechanical Actuators. With further development it could be used for condition monitoring and fault detection. That will be the goal of our investigation.

\section{References}

[1] Luenberger, D.G.: An Introduction to Observers, IEEE Transaction on Automatic Control, vol. AC-16, no. 6, 1971

[2] Kailath, T.: Linear Systems, Prentice Hall, pp. 79-152, 1980

[3] Reichhartinger, M., Horn, M.: Robust Position Control of an Electromechanical Actuator for Automotive Applications, World Academy of Science, Engineering and Technology, vol. 5, no. 11, pp. 1184-1188, 2011

[4] Lunze, J.: Regelungstechnik 2, Springer, pp. 337-366, 2010

[5] Padmakumar, S., Vivek A.: A Comparative Study into Observer based Fault Detection and Diagnosis in DC Motors: Part-I, World Academy of Science, Engineering and Technology, vol. 3, no. 3, pp. 294-299, 2009

[6] Grepl, R., Lee, B.: Modelling, Identification and Control of Electronics Throttle Body Using Dspace Tools, International Conference Technical Computing Prague, CD proceeding, pp. 1-10, 2008 
[7] Vašak, M., Petrović, I., Perić, N.: State Estimation of an Electronic Throttle Body, IEEE International Conference, vol. 1, pp. 472-477, 2003

DOI: 10.1109/ICIT.2003.1290368

[8] Nakano, K., Sawut, U., Higuci, K., Okajima, Y.: Modelling an Observer-based Sliding-Mode Control of Electronic Throttle Systems, ECTI Transactions on Electrical Eng, Electronics, and Communications, vol. 4, no. 1, pp. 22-27, 2006

[9] Kwadzogah, R., Misra, D.: Simultaneous Identification of Friction and Transfer Function of a DC Servo Positioning System via Simulation, International Journal of Intelligent Control and Systems, vol. 18, no. 1, pp. 1016,2013

[10] Angermann, A., Beuschel, M., Rau, M., Wolfahrt, U.: Matlab ${ }^{\circledR}$-Simulink ${ }^{\circledR}$ Stateflow $^{\circledR}$, Oldenburg Verlag, pp. 173-177, 2007

[11] Reinelt, W., Lundquest C.: Observer Based Sensor Monitoring in Active Front Steering System using Explicit Sensor Faliure Modelling, $16^{\text {th }}$ IFAC World Congress, vol. 16, no. 1, pp. 1-6, 2005 